\title{
First-principles investigation of B- and N-doped fluorographene
}

\author{
O. Leenaerts, ${ }^{*}$ H. Sahin, ${ }^{\dagger}$ B. Partoens, ${ }^{\ddagger}$ and F. M. Peeters ${ }^{\S}$ \\ Universiteit Antwerpen, Departement Fysica, Groenenborgerlaan 171, B-2020 Antwerpen, Belgium
}

(Received 26 June 2013; published 19 July 2013)

\begin{abstract}
The effect of substitutional doping of fluorographene with boron and nitrogen atoms on its electronic and magnetic properties is investigated using first-principles calculations. It is found that boron dopants can be readily incorporated in the fluorographene crystal where they act as shallow acceptors and cause hole doping, but no changes in the magnetic properties are observed. Nitrogen dopants act as deep donors and give rise to a magnetic moment, but the resulting system becomes chemically unstable. These results are opposite to what was found for substitutional doping of graphane, i.e., hydrogenated graphene, in which case B substituents induce magnetism and $\mathrm{N}$ dopants do not.
\end{abstract}

DOI: 10.1103/PhysRevB.88.035434

PACS number(s): 73.22.-f, 68.43.-h, 81.05.U-, 71.55.-i

\section{INTRODUCTION}

Although graphene is a very interesting material by itself, ${ }^{1}$ much research has been devoted to altering its physical and chemical properties by chemical functionalization. Some of the reasons for doing this are the absence of a band gap in the electronic spectrum of pristine graphene and the chemical inertness of its surface. The first property hinders the integration of graphene in traditional electronic devices while the second restricts its use in sensor applications. Chemical functionalization is an attractive way to solve these problems. The possibility to functionalize graphene with radicals such as $\mathrm{O}, \mathrm{F}$, and $\mathrm{H}$ atoms has been experimentally demonstrated and it was found that new crystalline materials, known as graphane (HG) and fluorographene or graphene fluoride (FG), are formed in the case of hydrogenation ${ }^{2,3}$ and fluorination, ${ }^{4,5}$ respectively. Oxidation of graphene, on the other hand, leads to an amorphous structure known as graphene oxide (GO) ${ }^{6,7}$ In addition, synthesis of chlorinated graphene and the existence of stable chlorographene $(\mathrm{ClG})$ structures were reported recently. ${ }^{8,9}$ All these functionalized materials are semiconductors which largely retain their strength and structural stability, ${ }^{10,11}$ although their crystal quality is appreciably reduced (in the case of $\mathrm{HG}$ and FG) or even completely destroyed (for GO). Similarly as in the case of pristine graphene, point defects can cause a magnetic response in graphane and fluorographene. It has been theoretically predicted and experimentally observed ${ }^{12}$ that a vacancy defect ${ }^{13}$ or covalently bonded adatoms ${ }^{14}$ can induce magnetism in graphene. Magnetism has also been predicted for $\mathrm{H}$ vacancies in graphane $\mathrm{e}^{15,16}$ and $\mathrm{F}$ vacancies in fluorographene, ${ }^{17}$ and it has been observed in fluorinated graphene samples. ${ }^{12}$ This magnetism is caused by the presence of unpaired electrons at the defect sites which can align anti- or ferromagnetically. Substitutional doping might also cause magnetism because it gives rise to unpaired electrons. However, the most common dopant atoms for carbon materials, boron and nitrogen, result only in charge doping of graphene and do not alter its magnetic properties. B-doped graphane, on the other hand, was predicted to be magnetic. ${ }^{18}$ In addition to possible charge doping and inducing a magnetic response, substitutional doping causes some carbon-based materials to become superconductors. This has been experimentally demonstrated for B-doped diamond ${ }^{19}$ and was recently predicted for B-doped graphane. ${ }^{20}$ In this work, we investigate the properties of substitutionally doped fluorographene. We only consider boron and nitrogen dopants because these form the most stable defects and they can be readily incorporated in a graphene lattice. The $\mathrm{N}$ (B) dopants add (remove) one electron to (from) carbon-based materials which leads to electron (hole) doping. Other dopants, such as $\mathrm{Al}, \mathrm{S}$, or P, are also possible, but these are much less stable and doping with these atoms is, consequently, more difficult to achieve. Since N- and B-doped graphene have already been realized ${ }^{21}$ subsequent fluorination of these doped graphene samples appears to be a feasible route to substitutionally doped fluorographene.

\section{COMPUTATIONAL DETAILS}

Our ab initio calculations are performed within the density functional theory formalism as implemented in the VASP simulation package. ${ }^{22,23}$ The simulations are done in a supercell geometry with sizes ranging from $2 \times 2$ to $6 \times 6$ unit cells (up to 144 atoms). We make use of the projector augmented wave method ${ }^{24-26}$ and a plane wave basis set with a cutoff energy of $500 \mathrm{eV}$. The generalized gradient approximation of Perdew, Burke, and Ernzerhof ${ }^{27}$ (GGA-PBE) is used for the exchange-correlation functional and spin polarization is included where necessary. The Brillouin zone sampling is done with a uniform $k$-point grid equivalent to a $24 \times 24 \times 1$ grid for a fluorographene unit cell. To avoid artificial interactions between periodic images we include a vacuum layer of $15 \AA$ in the simulations. We also perform ab initio molecular dynamics (MD) simulations in a $3 \times 3$ supercell to study the chemical stability of fluorine atoms adsorbed at the defect sites. The spin-polarized GGA-PBE exchange-correlation functional was also used in this case but the accuracy was reduced to make the simulations more manageable: The energy cutoff of the plane wave basis was lowered to $400 \mathrm{eV}$ and the sampling of the Brillouin zone was done with the equivalent of a $12 \times 12 \times 1 k$-point grid for a single FG unit cell. The integration of Newton's equations of motion was performed with the Verlet algorithm where Harris corrections are used in order to correct the forces. The simulations are performed within the microcanonical (NVE) ensemble with velocities assigned according to the Maxwell-Boltzmann distribution at a temperature of $300 \mathrm{~K}$. 
The stability of B-and N-doped fluorographene structures is also examined using phonon calculations. These are performed in the harmonic approximation by making use of the small displacement method implemented in the PHON code. ${ }^{28}$ The phonon dispersions and thermodynamic quantities of $3 \times$ 3 supercell structures of doped FG are calculated with a force field induced by a $0.01 \AA$ displacement. An atomic configuration is considered to be stable, if all the phonon branches have positive eigenfrequencies for all $q$ points in the Brillouin zone.

\section{RESULTS}

Before we investigate doped fluorographene, we study, as a first step, substitutional $\mathrm{B}$ and $\mathrm{N}$ doping in nonfluorinated graphene. We examined different doping concentrations ranging from 1.4 to 12.5 at. \%, corresponding to one dopant atom in a $6 \times 6$ and $2 \times 2$ graphene supercell, respectively. We define the formation energy of the substitutional defect as $E_{\mathrm{DF}}^{\prime}(\mathrm{X})=E_{\mathrm{G}+\mathrm{X}}-E_{\mathrm{G}}-E_{\mathrm{X}}+E_{\mathrm{C}}$, in which $E_{\mathrm{G}+\mathrm{X}}, E_{\mathrm{G}}, E_{\mathrm{X}}$, and $E_{\mathrm{C}}$ denote the energy of the doped graphene system, the corresponding pure graphene layer, and the (non-spinpolarized) atomic energies of the dopant and carbon atoms, respectively. The resulting formation energies are given in Table I. The sizes of $E_{\mathrm{DF}}^{\prime}$ for $\mathrm{N}$ and $\mathrm{B}$ doping are $E_{\mathrm{DF}}^{\prime}(\mathrm{N}) \approx$ $1.8 \mathrm{eV}$ and $E_{\mathrm{DF}}^{\prime}(\mathrm{B}) \approx 3.6 \mathrm{eV}$, respectively, which indicates that $\mathrm{N}$ doping in graphene is more favorable than $\mathrm{B}$ doping. The reason for this is structural: The $\mathrm{C}-\mathrm{N}$ bonds have a length of $\pm 1.41 \AA$ and fit nicely into the hexagonal carbon network $\left(d_{\mathrm{CC}}=1.425 \AA\right.$ ) whereas the $\mathrm{C}$-B bonds have lengths of $\pm 1.49 \AA$ and distort the graphene lattice. One can also observe a tendency of increasing formation energies with the concentration of dopants in both cases.

The addition or removal of one electron from the $\pi$-bonding electron network by the $\mathrm{N}$ and $\mathrm{B}$ doping gives rise to charge doping but does not induce any magnetism in the graphene crystal. This is in accordance with previous calculations and experiment. $^{29}$

Let us now turn our attention to doped fluorinated graphene. During fluorination, the doped graphene layers are covered with $\mathrm{F}$ atoms in such a way that every $\mathrm{C}$ atom, and also the dopant atoms, are bonded to $\mathrm{F}$ atoms above and below the graphene plane in an alternating pattern (chair configuration), as shown in Fig. 1.

In order to understand the stability of the resulting materials some additional energy-related quantities are defined.

TABLE I. The formation energy $E_{\mathrm{DF}}^{\prime}$ of substitutionally doped graphene and the dopant-carbon bond length $d_{\mathrm{CX}}(X=N, B)$ for different concentrations (i.e., supercell size). The energies are given in $\mathrm{eV}$ and the distances in $\AA$.

\begin{tabular}{llllll}
\hline \hline & $2 \times 2$ & $3 \times 3$ & $4 \times 4$ & $5 \times 5$ & $6 \times 6$ \\
\hline \multicolumn{7}{c}{ N doped } \\
$E_{\mathrm{DF}}^{\prime}$ & 1.953 & 1.884 & 1.789 & 1.761 & 1.758 \\
$d_{\mathrm{CN}}$ & 1.416 & 1.413 & 1.411 & 1.412 & 1.412 \\
\multicolumn{7}{c}{ B doped } \\
$E_{\mathrm{DF}}^{\prime}$ & 3.824 & 3.704 & 3.585 & 3.554 & 3.550 \\
$d_{\mathrm{CB}}$ & 1.498 & 1.494 & 1.494 & 1.493 & 1.492 \\
\hline \hline
\end{tabular}

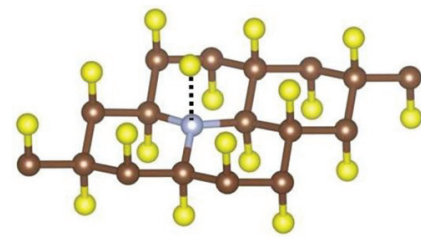

(a) N doped

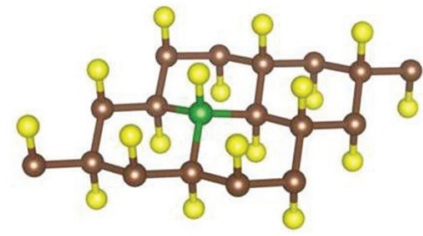

(b) B doped
FIG. 1. (Color online) A $3 \times 3$ FG supercell with one substitutional dopant atom: (a) $\mathrm{N}$ and (b) $\mathrm{B}$.

First we define the formation energy of the substitutional defect in FG similarly to our definition for graphene, $E_{\mathrm{DF}}=$ $E_{\mathrm{FG}+\mathrm{X}}-E_{\mathrm{FG}}-E_{\mathrm{X}}+E_{\mathrm{C}}$, in which $E_{\mathrm{FG}}$ is the energy of the corresponding fluorographene layer. A comparison of the defect formation energy in fluorographene (given in Table II) to the corresponding energy in graphene (Table I) provides information about the (de)stabilization of the defect after fluorination. In the case of B doping, fluorination is observed to have a stabilizing effect; i.e., B atoms are more easily integrated in FG than in graphene. $\mathrm{N}$ dopant atoms, however, are destabilized upon fluorination and are found to be less stable than B dopants. The reason is rather chemical than structural in this case. The extra electron on the $\mathrm{N}$ atom has to be placed in an antibonding orbital between the $\mathrm{N}$ and $\mathrm{F}$ atoms which makes this covalent bond unstable. A typical covalent $\mathrm{N}-\mathrm{F}$ bond (such as in $\mathrm{NF}_{3}$ molecules) is about $1.4 \AA$. As can be seen from the increased distance between $\mathrm{N}$ and $\mathrm{F}$ in $\mathrm{N}$-doped FG $\left(d_{\mathrm{NF}} \approx 2.1 \AA\right)$, this bond acquires a more ionic character. In order to examine this in further detail we define the binding energy $E_{b}$ as the energy to remove the $\mathrm{F}$ atom from the dopant site to infinity. It is seen from Table II that the binding energy for the $\mathrm{F}$ atom on the $\mathrm{N}$ dopant $\left(E_{\mathrm{b}} \approx-0.56 \mathrm{eV}\right)$ is much smaller than for the B dopant $\left(E_{\mathrm{b}} \approx-4.1 \mathrm{eV}\right)$, indicating the different type of bonding (ionic vs covalent).

For B dopants the formation energy tends to decrease with increasing concentration which is opposite to what we observed in graphene. But this does not imply that highly substitutionally doped FG samples are more stable. The formation energy of the defects decreases only slightly and remains always positive. Therefore, every additional defect

TABLE II. The formation energy $E_{\mathrm{DF}}$ and the binding energy $E_{\mathrm{b}}$ of substitutionally doped fluorographene for different concentrations (i.e., supercell size). The carbon-dopant $\left(d_{\mathrm{CX}}\right)$ and dopant-fluorine $\left(d_{\mathrm{XF}}\right)$ bond lengths are also given. The energies are given in $\mathrm{eV}$ and the distances in $\AA$.

\begin{tabular}{lrrrrr}
\hline \hline & $2 \times 2$ & $3 \times 3$ & $4 \times 4$ & $5 \times 5$ & $6 \times 6$ \\
\hline \multicolumn{5}{c}{$\mathrm{N}$ doped } \\
$E_{\mathrm{DF}}$ & 3.524 & 3.473 & 3.475 & 3.496 & 3.501 \\
$E_{\mathrm{b}}$ & -0.559 & -0.566 & -0.563 & -0.550 & -0.569 \\
$d_{\mathrm{CN}}$ & 1.512 & 1.509 & 1.508 & 1.508 & 1.508 \\
$d_{\mathrm{NF}}$ & 2.077 & 2.068 & 2.068 & 2.069 & 2.063 \\
& 1.798 & 1.939 & 2.022 & 2.068 & 2.092 \\
$E_{\mathrm{DF}}$ & -4.446 & -4.201 & -4.107 & -4.074 & -4.043 \\
$E_{\mathrm{b}}$ & 1.697 & 1.676 & 1.674 & 1.673 & 1.672 \\
$d_{\mathrm{CB}}$ & 1.368 & 1.376 & 1.378 & 1.379 & 1.379 \\
$d_{\mathrm{BF}}$ & 1.376 & \\
\hline \hline
\end{tabular}




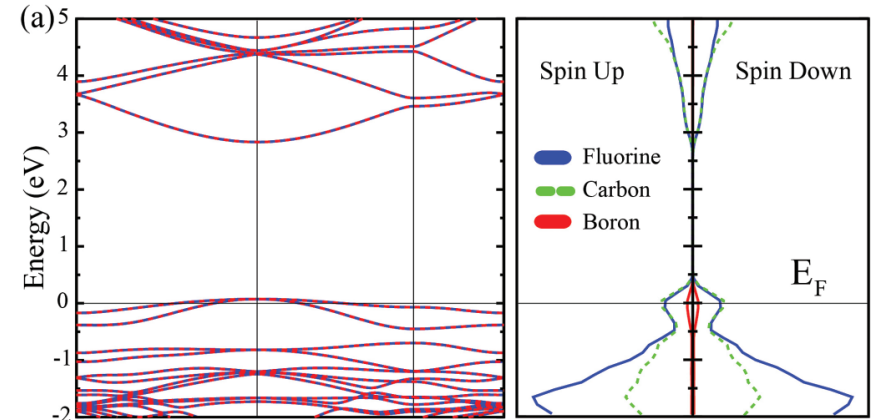

(b)

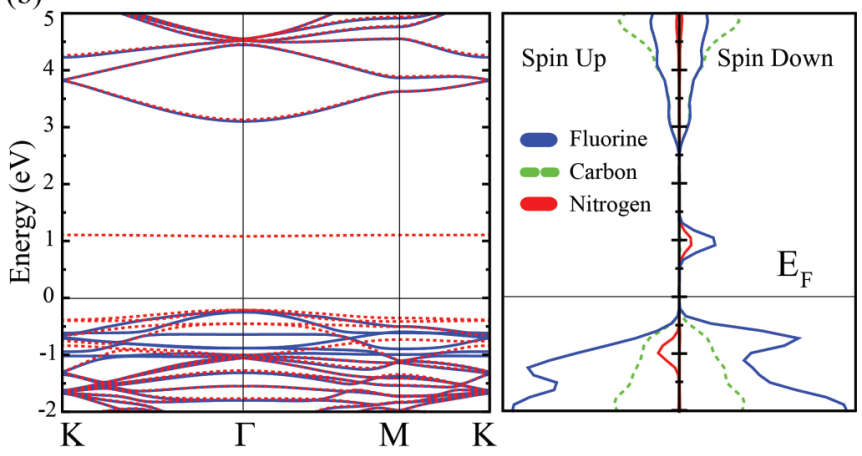

FIG. 2. (Color online) The spin-polarized electronic band structure and projected density of states of B (a) and N (b) doped fluorographene calculated for a $4 \times 4$ supercell. Spin up and down states are shown by blue solid and red dashed lines, respectively. The Fermi level is set to zero.

will decrease the stability of the system. For $\mathrm{N}$ doping, the dependence of the formation energy on the dopant concentration is not monotonic because of the interaction of the magnetic moments in neighboring supercells at higher concentrations.

Let us now take a closer look at the magnetic properties of these doped FG materials. It was shown above that substitutional doping in graphene does not induce any magnetism. Magnetism is also absent after fluorination of a B-doped graphene layer for any concentration of dopants. In the case of $\mathrm{N}$ doping, on the other hand, every dopant induces a magnetic moment of $\mu_{b}$ in fluorographene, independent of the concentration, and this magnetic moment is mainly located on the ionically bonded $\mathrm{F}$ atom. We can understand this by looking at the $\mathrm{N}$-doped fluorographene without an adsorbed $\mathrm{F}$ atom at the defect site, which is nonmagnetic (see below), and an isolated $\mathrm{F}$ atom which has a magnetic moment of $1 \mu_{b}$. The weak interaction between these two systems does not delocalize the magnetic moment so it remains mainly localized on the $\mathrm{F}$ atom.

It is interesting to note that the magnetic properties that we found for B- and N-doped fluorographene are opposite to substitutionally doped graphane (i.e., hydrogenated doped graphene), where B doping leads to magnetism while $\mathrm{N}$ doping does not. $^{18}$

One of the most important reasons for doping fluorographene with foreign atoms is the possibility of creating charge carriers in this semiconducting material. The additional or missing electron in the case of $\mathrm{N}$ and $\mathrm{B}$ doping, respectively, can cause partially occupied electronic bands which can induce a semiconductor-to-metal transition. This is, e.g., the case for N-, B-, and P-doped graphane, which are predicted to be metallic, and Al-doped graphane which forms a half metal. ${ }^{18}$ In Fig. 2 we show the (spin-polarized) electronic band structure for $\mathrm{N}$ - and B-doped fluorographene. B-doped FG is found to be metallic because the Fermi-level is shifted into the valence bands of FG due to the missing electron. The situation is very different for $\mathrm{N}$-doped FG where no metallic behavior is observed. In this case a (nondispersive) defect band is created which is split into a spin up and spin down band. One of these bands lies below the Fermi-level and the other is located inside the band gap and remains unoccupied, as can be clearly seen in the projected density of states (PDOS) of N-doped FG [Fig. 2(b)].

Now that we have studied the electronic and magnetic properties of B- and $\mathrm{N}$-doped FG, we will focus in more detail on the stability of these doped FG systems. In particular, we will examine their dynamical and chemical stability. While energy optimizations as obtained above imply the optimizability of a given atomic configuration, the dynamical stability of a system can only be understood through its phonon spectrum.

In Fig. 3 we show the phonon frequency dispersions and the phonon density of states of pure FG, B-doped FG, and $\mathrm{N}$-doped FG. Pure FG has 12 characteristic eigenmodes: three acoustic modes (LA, TA, and ZA), four phonon modes around 250 and $300 \mathrm{~cm}^{-1}$ that correspond to in-plane motion of $\mathrm{C}$ and $\mathrm{F}$ atoms, a breathing mode at $710 \mathrm{~cm}^{-1}$, and four optical modes between $1230-1300 \mathrm{~cm}^{-1}$ that correspond to out-of-plane motion of $\mathrm{C}$ and $\mathrm{F}$ atoms. Phonon frequency dispersions shown in Fig. 3 indicate that there is no dopant-induced instability and hence confirm the dynamical stability of both $\mathrm{B}$ - and $\mathrm{N}$-doped fluorographene. Note that the small negative values of the out-of-plane acoustic (ZA) branch stems from the discreteness of FFT grid and therefore this artificially imaginary eigenfrequency is not an indication of any real instability.

It is seen that there is no significant change in acoustical phonon frequencies upon $\mathrm{N}$ and $\mathrm{B}$ doping and that dopantrelated modes appear in optical branches. The dispersion of B-related phonon branches can be characterized by two main regions: a low-energy region at $490 \mathrm{~cm}^{-1}$ characterized by mixing of in-plane motion of $\mathrm{C}, \mathrm{B}$, and $\mathrm{F}$ atoms and a highenergy region at $1140 \mathrm{~cm}^{-1}$ characterized by collective outof-plane motion of dopant and FG. However, the dispersion of N-doped FG shows more localized behavior because of the larger atomic mass of the $\mathrm{N}$ atom and two main $\mathrm{N}$-related peaks in the density of states are visible. While the mode at $725 \mathrm{~cm}^{-1}$ is related to the collective breathing motion of $\mathrm{N}$ and FG, the phonon mode at $868 \mathrm{~cm}^{-1}$ is a combination of the dopant's out-of-plane motion and the in-plane motion of FG.

A common characteristic of both $\mathrm{B}$ and $\mathrm{N}$ doping is a splitting that occurs between the high-energy optical phonon band of FG and the optical branches lower than $1000 \mathrm{~cm}^{-1}$. Though the bands are split, $\mathrm{N}$ doping does not yield a gap in the phonon DOS. However the B-induced gap in the phonon DOS is $5 \mathrm{~cm}^{-1}$. Another characteristic of $\mathrm{B}$ and $\mathrm{N}$ doping is the shift of the highest optical mode that corresponds to out-of-plane counterphase vibrational motion of $\mathrm{C}$ and $\mathrm{F}$ atoms, away from the low-lying optical modes. 
(a) Fluorographene

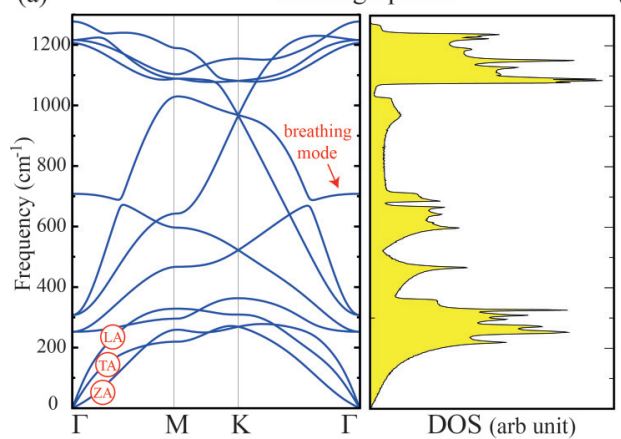

(b)

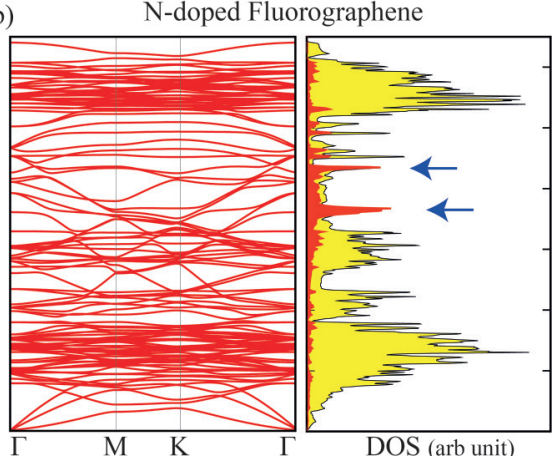

(c)

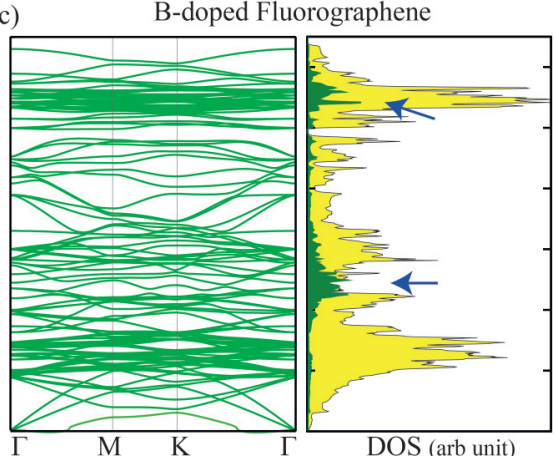

FIG. 3. (Color online) Phonon dispersions and partial density of states of undoped, B-doped, and N-doped FG. The total-DOS, N-DOS, and B-DOS are shown by yellow, red, and green colors, respectively. The arrows indicate phonon frequencies that are discussed in the text.

The phonon spectra of B- and N-doped FG prove the dynamical stability of these systems. The binding energies $\left(E_{b}\right)$ from Table II show that $\mathrm{F}$ atoms are weakly bonded to the $\mathrm{N}$ defect site $\left(E_{b} \approx-0.56 \mathrm{eV}\right)$, while they are strongly bonded at the $\mathrm{B}$ sites $\left(E_{b} \approx-4.1 \mathrm{eV}\right)$. These binding energies are large enough to ensure also thermal stability; i.e., the $\mathrm{F}$ atoms at the defect sites are stable against thermal desorption. The relatively small size of $E_{b}$ for $\mathrm{N}$ dopants, on the other hand, suggests that the chemical stability of this defect site is rather low.

To investigate this in further detail, we performed ab initio molecular dynamics (MD) simulations for this system. These MD simulations show that the loosely bonded $F$ atom is rather reactive and can easily bind to atoms or molecules in the environment. For example, approaching $\mathrm{H}$ and $\mathrm{F}$ atoms readily disrupt the weak bond to form respectively $\mathrm{HF}$ and $\mathrm{F}_{2}$ molecules which are easily detached from the surface. An interesting phenomenon is observed when a hydrogen molecule approaches the defect site. The interaction process is illustrated in Fig. 4.

In a first step the $\mathrm{H}_{2}$ molecule is attracted by the $\mathrm{F}$ atom and binds to it. Then the hydrogen bond is broken and a hydrogen fluoride molecule is formed from the $\mathrm{F}$ atom and one of the $\mathrm{H}$ atoms. If the kinetic energy of the other $\mathrm{H}$ atom is large

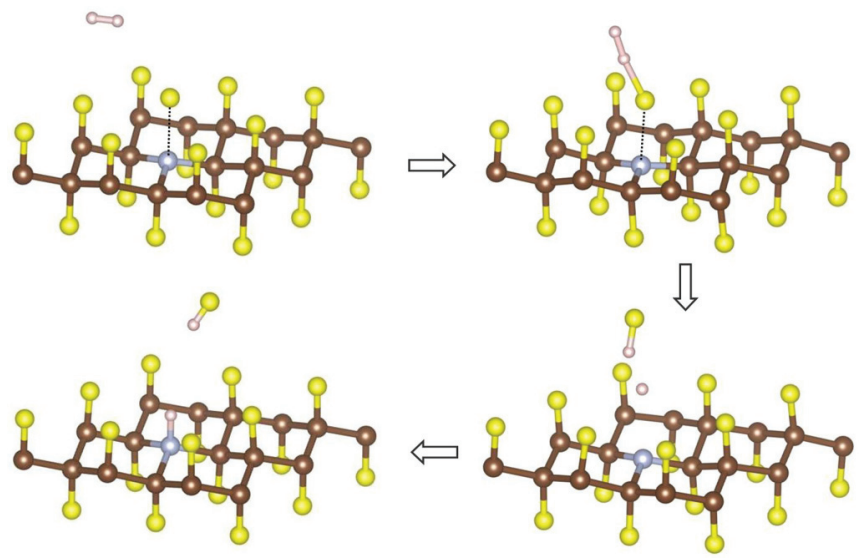

FIG. 4. (Color online) Different steps of the interaction process of a $\mathrm{H}_{2}$ molecule with $\mathrm{N}$-doped FG simulated with MD. The last step only occurs if the isolated $\mathrm{H}$ atom is given an additional kinetic energy of approximately $1 \mathrm{eV}$. enough, it can form a bond with the exposed $\mathrm{N}$ atom. This bond has a length of $1.036 \AA$ which is a typical size for a covalent $\mathrm{N}-\mathrm{H}$ bond. Contrary to the fully fluorinated doped FG system, the $\mathrm{N}$-doped FG layer with $\mathrm{H}$ substitutions at the dopant sites is nonmagnetic and metallic [see Fig. 5(b)] and would be a good candidate to achieve electron charge doping in FG. Unfortunately, the $\mathrm{H}$ substitutions at the defect sites are not very likely to occur because a reaction barrier of more than $1 \mathrm{eV}$ needs to be overcome for the N-H bonds to form. This can be explained from the electronic configuration of the $\mathrm{N}$ atoms: The $\mathrm{N}$ atom in $\mathrm{N}$-doped FG has $4 s p^{3}$ orbitals of which 3 are used to form covalent bonds to the neighboring $C$ atoms. Three of the five valence electrons of the $\mathrm{N}$ atom are used in these bonds while the other two are placed in the fourth orbital which is therefore fully occupied. Although it is still possible to use this fourth orbital to form weak bonds with the

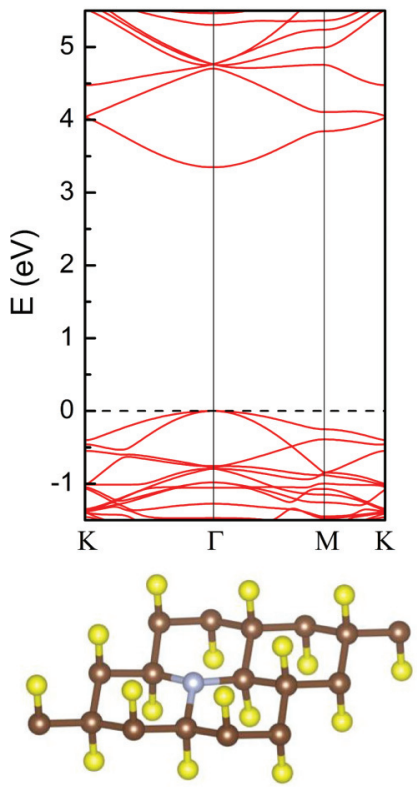

(a) missing $\mathrm{F}$

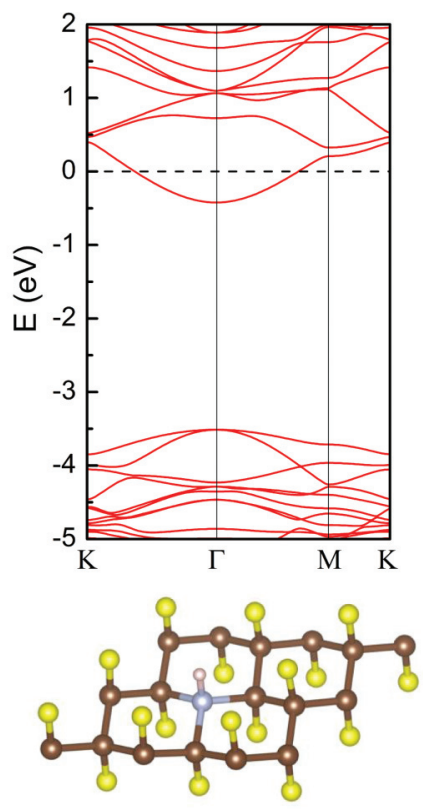

(b) substituted $\mathrm{H}$
FIG. 5. (Color online) The electronic band structure (top) and a visualization (bottom) of $\mathrm{N}$-doped $\mathrm{FG}$ with one missing $\mathrm{F}$ atom (a) and the system with the $\mathrm{F}$ atom at the dopant site substituted by $\mathrm{H}(\mathrm{b})$. 
$\mathrm{N}$ atom (as in the case of $\mathrm{H}$ or $\mathrm{F}$ atoms), this is energetically unfavorable and not very likely to happen. It appears therefore that no atoms will form bonds with the $\mathrm{N}$ atoms, so that the $\mathrm{N}$ dopant sites will probably remain unoccupied.

The electronic band structure of the N-doped FG with unoccupied defect sites, shown in Fig. 5(a), is almost indistinguishable of undoped fluorographene and $\mathrm{N}$ doping thus appears to be not a fruitful possibility to induce charge carriers or alter the electronic properties of FG.

\section{SUMMARY AND CONCLUSIONS}

We investigated the possibility of doping fluorographene with substitutional boron and nitrogen atoms. In comparison to substitutionally doped graphene, fluorination has a stabilizing effect on B dopants and a destabilizing effect on $\mathrm{N}$ substitutions. B dopants act as shallow acceptors and can be used to achieve hole doping in fluorographene. In contrast to B-doped graphane, B substitutions do not induce any magnetism in fluorographene. Nitrogen dopants act as deep donors and give rise to a magnetic moment upon full fluorination, but the resulting system becomes chemically unstable. We performed $a b$ initio molecular dynamic simulations to show that the fluorine atoms that are bonded to the $\mathrm{N}$ dopants can be easily removed by atoms or molecules (such as $\mathrm{H}_{2}$ ) in the environment. The removal of these $\mathrm{F}$ atoms cancels the electron doping resulting from the nitrogen atoms and, consequently, no charge carriers are present. Electron doping can be achieved by saturation of the vacant $F$ sites with $H$ atoms. These adsorbates are more strongly attached to the $\mathrm{N}$ dopant, but there is a large adsorption barrier $(>1 \mathrm{eV})$ for this process to occur and the resulting system is nonmagnetic.

To conclude, hole doping in fluorographene can be readily achieved by incorporating boron dopants into the system, while electron doping is more difficult to achieve.

\section{ACKNOWLEDGMENTS}

This work was supported by the Flemish Science Foundation (FWO-Vl) and the Methusalem program of the Flemish government. H.S. is supported by a FWO Pegasus-long Marie Curie Fellowship. Computational resources were provided by TUBITAK ULAKBIM, High Performance and Grid Computing Center (TR-Grid e-Infrastructure), and the HPC infrastructure of the University of Antwerp (CalcUA), a division of the Flemish Supercomputer Center VSC. *ortwin.leenaerts@ua.ac.be

†hasan.sahin@ua.ac.be

†bart.partoens@ua.ac.be

§rancois.peeters@ua.ac.be

${ }^{1}$ K. S. Novoselov, A. K. Geim, S. V. Morozov, D. Jiang, Y. Zhang, S. V. Dubonos, I. V. Grigorieva, and A. A. Firsov, Science 306, 666 (2004).

${ }^{2}$ D. C. Elias, R. R. Nair, T. M. G. Mohiuddin, S. V. Morozov, P. Blake, M. P. Halsall, A. C. Ferrari, D. W. Boukhvalov, M. I. Katsnelson, A. K. Geim, and K. S. Novoselov, Science 323, 610 (2009).

${ }^{3}$ S. H. Cheng, K. Zou, F. Okino, H. R. Gutierrez, A. Gupta, N. Shen, P. C. Eklund, J. O. Sofo, and J. Zhu, Phys. Rev. B 81, 205435 (2010).

${ }^{4}$ F. Withers, M. Dubois, and A. K. Savchenko, Phys. Rev. B 82, 073403 (2010).

${ }^{5}$ R. R. Nair, W. C. Ren, R. Jalil, I. Riaz, V. G. Kravets, L. Britnell, P. Blake, F. Schedin, A. S. Mayorov, S. Yuan, M. I. Katsnelson, H. M. Cheng, W. Strupinski, L. G. Bulusheva, A. V. Okotrub, I. V. Grigorieva, A. N. Grigorenko, K. S. Novoselov, and A. K. Geim, Small 6, 2877 (2010).

${ }^{6}$ D. A. Dikin, S. Stankovich, E. J. Zimney, R. D. Piner, G. H. B. Dommett, G. Evmenenko, S. T. Nguyen, and R. S. Ruoff, Nature (London) 448, 457 (2007).

${ }^{7}$ G. Eda and M. Chhowalla, Adv. Mater. 22, 2392 (2010).

${ }^{8}$ H. Sahin and S. Ciraci, J. Phys. Chem. C 116, 24075 (2012).

${ }^{9}$ B. Li, L. Zhou, D. Wu, H. Peng, K. Yan, Y. Zhou, and Z. Liu, ACS Nano. 5, 5957 (2011).

${ }^{10}$ J. O. Sofo, A. S. Chaudhari, and G. D. Barber, Phys. Rev. B 75, 153401 (2007).

${ }^{11}$ O. Leenaerts, H. Peelaers, A. D. Hernández-Nieves, B. Partoens, and F. M. Peeters, Phys. Rev. B 82, 195436 (2010).

${ }^{12}$ R. R. Nair, M. Sepioni, I-Ling Tsai, O. Lehtinen, J. Keinonen, A. V. Krasheninnikov, T. Thomson, A. K. Geim, and I. V. Grigorieva, Nat. Phys. 8, 199 (2012).
${ }^{13}$ J. J. Palacios, J. Fernández-Rossier, and L. Brey, Phys. Rev. B 77, 195428 (2008)

${ }^{14}$ W. Li, M. Zhao, Y. Xia, R. Zhang, and Y. Mu, J. Mater. Chem. 19, 9274 (2009).

${ }^{15}$ H. Sahin, C. Ataca, and S. Ciraci, Appl. Phys. Lett. 95, 222510 (2009).

${ }^{16}$ J. Berashevich and T. Chakraborty, Nanotechnology 21, 355201 (2010).

${ }^{17}$ H. Sahin, M. Topsakal, and S. Ciraci, Phys. Rev. B 83, 115432 (2011).

${ }^{18}$ Y. Wan, Y. Ding, S. Shi, and W. Tang, Appl. Phys. Lett. 98, 163104 (2011).

${ }^{19}$ E. A. Ekimov, V. A. Sidorov, E. D. Bauer, N. N. Mel'nik, N. J. Curro, J. D. Thompson, and S. M. Stishov, Nature (London) 428 , 542 (2004).

${ }^{20}$ G. Savini, A. C. Ferrari, and F. Giustino, Phys. Rev. Lett. 105, 037002 (2010).

${ }^{21}$ L. S. Panchakarla, K. S. Subrahmanyam, S. K. Saha, Achutharao Govindaraj, H. R. Krishnamurthy, U. V. Waghmare, and C. N. R. Rao, Adv. Mater. 21, 4726 (2009).

${ }^{22}$ G. Kresse and J. Furthmüller, Comput. Mater. Sci. 6, 15 (1996).

${ }^{23}$ G. Kresse and J. Furthmüller, Phys. Rev. B 54, 11169 (1996).

${ }^{24}$ P. E. Blöchl, Phys. Rev. B 50, 17953 (1994).

${ }^{25} \mathrm{G}$. Kresse and D. Joubert, Phys. Rev. B 59, 1758 (1999).

${ }^{26}$ B. Adolph, J. Furthmüller, and F. Bechstedt, Phys. Rev. B 63, 125108 (2001).

${ }^{27}$ J. P. Perdew, K. Burke, and M. Ernzerhof, Phys. Rev. Lett. 77, 3865 (1996).

${ }^{28}$ D. Alfe, Comput. Phys. Commun. 180, 2622 (2009).

${ }^{29}$ H. Liu, Y. Liu, and D. Zhu, J. Mater. Chem. 21, 3335 (2011). 